\title{
Trivial, Critical and Near-critical Scaling Limits of Two-dimensional Percolation
}

\author{
Federico Camia $\cdot$ Matthijs Joosten $\cdot$ Ronald Meester
}

Received: 17 April 2009 / Accepted: 22 September 2009 / Published online: 8 October 2009

(C) The Author(s) 2009. This article is published with open access at Springerlink.com

\begin{abstract}
It is natural to expect that there are only three possible types of scaling limits for the collection of all percolation interfaces in the plane: (1) a trivial one, consisting of no curves at all, (2) a critical one, in which all points of the plane are surrounded by arbitrarily large loops and every deterministic point is almost surely surrounded by a countably infinite family of nested loops with radii going to zero, and (3) an intermediate one, in which every deterministic point of the plane is almost surely surrounded by a largest loop and by a countably infinite family of nested loops with radii going to zero. We show how one can prove this using elementary arguments, with the help of known scaling relations for percolation.

The trivial limit corresponds to subcritical and supercritical percolation, as well as to the case when the density $p$ approaches the critical probability, $p_{c}$, sufficiently slowly as the lattice spacing is sent to zero. The second type corresponds to critical percolation and to a faster approach of $p$ to $p_{c}$. The third, or near-critical, type of limit corresponds to an intermediate speed of approach of $p$ to $p_{c}$. The fact that in the near-critical case a deterministic point is a.s. surrounded by a largest loop demonstrates the persistence of a macroscopic correlation length in the scaling limit and the absence of scale invariance.
\end{abstract}

Keywords Percolation · Continuum scaling limit $\cdot$ Near-critical regime $\cdot$ Massive scaling

Research of F. Camia supported in part by a Veni grant of the NWO (Dutch Organization for Scientific Research).

Research of R. Meester supported in part by a Vici grant of the NWO (Dutch Organization for Scientific Research).

F. Camia $(\bowtie) \cdot$ M. Joosten $\cdot$ R. Meester

Department of Mathematics, Vrije Universiteit, Amsterdam, The Netherlands

e-mail: fede@few.vu.nl

M. Joosten

e-mail: mjoosten@few.vu.nl

R. Meester

e-mail: rmeester@few.vu.nl 


\section{Introduction and Main Results}

In Bernoulli site (respectively, bond) percolation, the sites (resp., bonds) of a regular lattice with lattice spacing $\delta$ are colored white with probability $p$ and black otherwise, independently of each other. One is then interested in the connectivity properties of the monochromatic subgraphs of the lattice, called clusters (see, e.g., $[5,15,16]$ ).

The rigorous geometric analysis of the continuum scaling limit $(\delta \rightarrow 0)$ of two-dimensional critical site percolation on the triangular lattice has made tremendous progress in recent years. In particular, the work of Schramm [21] and Smirnov [22] has allowed to identify the scaling limit of critical interfaces (i.e., boundaries between black and white clusters) in terms of the Schramm-Loewner Evolution (SLE) (see also [11, 23]). Based on that, Camia and Newman have constructed [9] a process of continuum nonsimple loops in the plane, and proved [10] that it coincides with the scaling limit of the collection of all percolation interfaces (the full scaling limit). The use of SLE technology and computations, combined with Kesten's scaling relations [17], has also led to the derivation of important properties of percolation such as the values of some critical exponents [18, 24].

In later work [7, 8], based on heuristic arguments, Camia, Fontes and Newman have proposed an approach for obtaining a one-parameter family of near-critical scaling limits with density of white sites (or bonds) given by

$$
p=p_{c}+\lambda \delta^{\alpha}
$$

where $p_{c}$ is the critical density, $\delta$ is the lattice spacing, $\lambda \in(-\infty, \infty)$, and $\alpha$ is set equal to $3 / 4$ to get nontrivial $\lambda$-dependence in the limit $\delta \rightarrow 0$ (see below and $[1,2,6]$ ). The approach proposed in $[7,8]$ is based on the critical full scaling limit and the "Poissonian marking" of some special ("macroscopically pivotal") points, and it leads to a conceptual framework that can in principle describe not only the scaling limit of near-critical percolation but also of related two-dimensional models such as dynamical percolation, the minimal spanning tree and invasion percolation (see [8]).

In this note, we consider the collection of all percolation interfaces and show how one can use known scaling relations for percolation to prove that, besides the trivial scaling limit corresponding to subcritical and supercritical percolation, there are only two other alternatives, that we call critical and near-critical scaling limits, and for which we give a geometric characterization.

We postpone precise definitions till Sects. 2 and 3 (including those of the space of interfaces and the topology of weak convergence), but in order to avoid delaying the statement of the main result, we present it here. We denote by $P_{\delta, p}$ the probability measure corresponding to Bernoulli site percolation on the triangular lattice with lattice spacing $\delta$ and parameter $p$. It is well known [16] that percolation on the triangular lattice has a phase transition at $p=1 / 2$. Let $H_{\delta}^{w}(n)$ denote the event that there is a white horizontal crossing in a "box" of Euclidean side length $n \delta$ on the lattice with lattice spacing $\delta$ (see Fig. 2 and the next section for precise definitions). Due to the black/white symmetry of the model, without loss of generality, we can restrict our attention to the case $p \geq 1 / 2$. For $\epsilon \in(0,1 / 2)$, we define

$$
p_{\epsilon}^{+}(n):=\inf \left\{p: P_{\delta, p}\left(H_{\delta}^{w}(n)\right)>1 / 2+\epsilon\right\} .
$$

(Note that $p_{\epsilon}^{+}(n)$ is independent of $\delta, p_{\epsilon}^{+}(n) \geq 1 / 2 \forall \epsilon \in(0,1 / 2)$ and $p_{\epsilon_{1}}^{+}(n) \leq p_{\epsilon_{2}}^{+}(n)$ if $\epsilon_{1} \leq \epsilon_{2}$.) Let $\mu_{\delta, p}$ denote the distribution of all percolation interfaces for site percolation with parameter $p$ on the triangular lattice with lattice spacing $\delta$. 
Theorem 1.1 Suppose that $\mu$ is the weak limit of a sequence $\left\{\mu_{\delta_{j}, p_{j}}\right\}_{j \in \mathbb{N}}$, with $\delta_{j} \rightarrow 0$ as $j \rightarrow \infty$ and $p_{j} \geq 1 / 2$ for all $j$. Then one of the following non-void scenarios holds.

(1) Trivial scaling limit: $\mu$-a.s. there are no loops of diameter larger than zero.

(2) Critical scaling limit: $\mu$-a.s. any deterministic point in the plane is surrounded by a countably infinite family of nested loops with radii going to zero. Moreover, every point is surrounded by a countably infinite family of nested loops with radii going to infinity.

(3) Near-critical scaling limit: $\mu$-a.s. any deterministic point in the plane is surrounded by a largest loop and by a countably infinite family of nested loops with radii going to zero.

Moreover, the third scenario can be realized by taking $0<\epsilon_{1}<\epsilon_{2}<1 / 2$ and (an appropriate subsequence of) $\left\{p_{j}\right\}_{j \in \mathbb{N}}$ chosen so that $p_{\epsilon_{1}}^{+}\left(1 / \delta_{j}\right) \leq p_{j} \leq p_{\epsilon_{2}}^{+}\left(1 / \delta_{j}\right)$ for every $j$.

The above geometric characterization of near-critical scaling limits, case (3), was conjectured in [7]. It shows that such limits are not scale invariant and differ qualitatively from the critical scaling limit at large scales, since in the latter case there is no largest loop around any point. At the same time, they resemble the critical scaling limit at short scales because of the presence, around any given point, of infinitely many nested loops with radii going to zero. Depending on the context, this situation is also described as off-critical or massive scaling limit (where "massive" refers to the persistence of a macroscopic correlation length, which should give rise to what is known in the physics literature as a "massive field theory").

The three regimes in Theorem 1.1 correspond to those in Proposition 4 of [20], which contains, among other things, results analogous to some of ours in the context of a single percolation interface and its scaling limit. Perhaps the most interesting results of [20] and of this paper concern the near-critical regime (regime (3) of Theorem 1.1). While [20] deals with a single interface, proving that its scaling limit in the near-critical regime is singular with respect to $\mathrm{SLE}_{6}$ (the critical scaling limit), in this paper we consider the full scaling limit and are concerned with the geometry of the set of all interfaces, so that, in some sense, our result on the near-critical regime complements that of [20].

Our results imply that when $\alpha>3 / 4$ in (1), the full scaling limit is trivial, when $\alpha<3 / 4$ it is critical, and there is a non-empty regime where it is neither trivial nor critical. The following corollary is an immediate consequence of Theorem 1.1 and the power law (2) given at the end of the next section.

Corollary 1.2 Consider site percolation on the triangular lattice with lattice spacing $\delta$ and parameter $p=1 / 2+\lambda \delta^{\alpha}$. Then, for every $\lambda \in(-\infty, \infty)$,

- if $\alpha<3 / 4$, there is a unique scaling limit which is trivial in the sense of Theorem 1.1 ,

- if $\alpha>3 / 4$, every subsequential scaling limit is critical in the sense of Theorem 1.1.

It is natural to conjecture that the near-critical regime (case (3) in Theorem 1.1) corresponds to the case $\alpha=3 / 4$, but at the moment this is not known. In order to prove that, one would need to show that when $\alpha=3 / 4$ the correlation length $L_{\epsilon}(p)$ defined in Sect. 2 below remains bounded away from zero and infinity as $\delta \rightarrow 0$ (see the proof of case (3) of Theorem 1.1). This is believed to be the case, and in fact the correlation length is expected to follow the power law $L_{\epsilon}(p) \asymp|p-1 / 2|^{-4 / 3}$ as $p \rightarrow 1 / 2$, where $\asymp$ means that the ratio between the two quantities is bounded away from zero and infinity. However, only the weaker power law $L_{\epsilon}(p)=|p-1 / 2|^{-4 / 3+o(1)}$ has been proved [24]. 
In a remark at the end of Sect. 4, we explain how one can combine [10] with (part of) the proof of Proposition 4 of [20] to obtain a (much) stronger version of case (2) of Theorem 1.1 (namely, that the scaling limit in regime (2) coincides with the critical full scaling limit $[9,10])$. In view of this result, in the second item of Corollary 1.2, one can identify the scaling limit with $\alpha>3 / 4$ with the unique critical scaling limit $[9,10]$.

It is our understanding that significant progress has recently been made [14] (see also [13]) in proving the approach of [7, 8] to near-critical scaling limits. A consequence would be that all subsequential limits discussed in this paper are in fact limits.

To conclude this section, we point out that, although our results are stated for site percolation on the triangular lattice, except for Corollary 1.2 which relies on the power law (2) and Remark 4.1 which relies on results from $[10,11,20]$, they also apply to bond percolation and to other regular lattices like the square lattice (after replacing $1 / 2$ with $p_{c}$ when necessary). Indeed, the main tools in our proofs originated in Kesten's work [17] on the square lattice and can be used for both site and bond percolation models on a large class of lattices (see [16]). For a discussion of the range of applicability of Kesten's and related results, and consequently of the results of the present paper, the reader is referred to Sect. 8.1 of [19].

\section{Notation and Some Background}

Consider the hexagonal lattice $\mathcal{H}_{\delta}$ with lattice spacing $\delta>0$, and its dual, the triangular lattice $\mathcal{T}_{\delta}$, embedded in $\mathbb{R}^{2}$ as in Fig. 1 . A site of the triangular lattice is identified with the face of the hexagonal lattice that contains it.

Throughout this paper, we are interested in Bernoulli site percolation on $\mathcal{T}_{\delta}$, defined as follows. Each site of $\mathcal{T}_{\delta}$ is independently declared white, and the corresponding hexagon colored white, with probability $p$. Sites that are not white are declared black, and the corresponding hexagons are colored black. We denote by $P_{\delta, p}$ the probability measure corresponding to site percolation on $\mathcal{T}_{\delta}$ with parameter $p$. It is well known [16] that percolation on the triangular lattice has a phase transition at $p=1 / 2$.

A path of length $n$ in $\mathcal{T}_{\delta}$ is a sequence of $n$ distinct sites $\left(x_{1}, x_{2}, \ldots, x_{n}\right)$ of $\mathcal{T}_{\delta}$ and the edges of $\mathcal{T}_{\delta}$ between them such that $x_{k}$ and $x_{k+1}$ are adjacent in $\mathcal{T}_{\delta}$ for all $k=1, \ldots, n-1$. A circuit of length $n$ is a path whose first and last sites are adjacent. We define the diameter of a set $U \subset \mathbb{R}^{2}$ as

$$
\operatorname{diam}(U):=\sup \{|x-y|: x, y \in U\},
$$

where $|\cdot|$ denotes Euclidean distance. We call a path or a circuit white (resp., black) if all its sites are white (resp., black).

The edges between neighboring hexagons with different colors form interfaces. A concatenation of such edges will be called a boundary path or a boundary loop if it forms a

Fig. 1 Embedding of the triangular and hexagonal lattices in $\mathbb{R}^{2}$

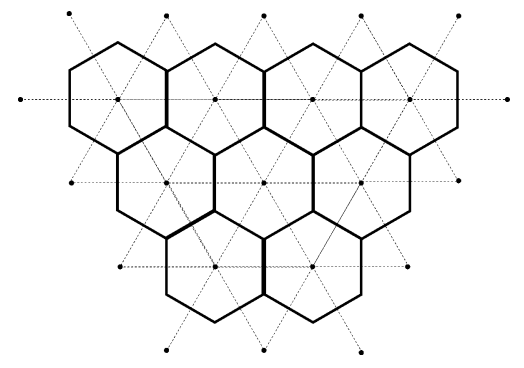


Fig. 2 The box $B(x ; r)$

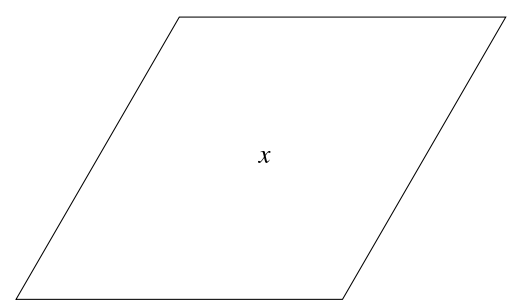

closed curve. Note that boundary curves and loops are always simple (i.e., no self-touching occurs) for $\delta>0$. However, this will not necessarily be the case in the scaling limit $\delta \rightarrow 0$.

For $n_{1}, n_{2}>0,\left[0, n_{1}\right] \times\left[0, n_{2}\right]$ will denote the closed parallelogram with Euclidean sidelengths $n_{1}$ and $n_{2}$ and sides which are parallel to two of the axes of the triangular lattice as in Fig. 2. In particular, when $n_{1}=n_{2}$, we call such a parallelogram a box. $B(x ; r)$ will denote the box centered at $x$, obtained by translating $[0, r] \times[0, r]$ (see Fig. 2). For $0<r<R$, we define the annulus $A(x ; r, R)$ as

$$
A(x ; r, R):=B(x ; R) \backslash B^{\circ}(x ; r),
$$

where $B^{\circ}(x ; r)$ denotes the interior of $B(x ; r)$. When $x$ is the origin, we will write $B(r)$ and $A(r, R)$, respectively. Note that boxes and annuli are defined in terms of the Euclidean metric and not relative to the lattice spacing.

The notion of correlation length will be very important. Various equivalent definitions are possible; we choose the one, introduced in [12] and also used in [17], that is most suitable for our purposes. Let $n$ be an integer and $H_{\delta}^{w}(n)$ be the event that a percolation configuration on $\mathcal{T}_{\delta}$ contains a white path inside $B(n \delta)$ intersecting both its "left side" and its "right side." For each $\epsilon \in(0,1 / 2)$, the correlation length $L_{\epsilon}(p)$ is defined as follows:

$$
\begin{aligned}
& L_{\epsilon}(p):=\min \left\{n: P_{\delta, p}\left(H_{\delta}^{w}(n)\right)>1 / 2+\epsilon\right\} \quad \text { when } p>1 / 2, \\
& L_{\epsilon}(p):=\min \left\{n: P_{\delta, p}\left(H_{\delta}^{w}(n)\right)<1 / 2-\epsilon\right\} \quad \text { when } p<1 / 2 .
\end{aligned}
$$

We also define $L_{\epsilon}(1 / 2)=\infty$ for all $\epsilon \in(0,1 / 2)$. Note that in the definition above, the correlation length is measured in lattice spacings (rather than in the Euclidean metric), and is therefore independent of $\delta$. Below we will frequently make use of the scaled correlation length $\delta L_{\epsilon}(p)$, which can be seen as the "macroscopic" correlation length.

An important fact about the correlation length is that the $\epsilon$ in the definition is unimportant, due to the following result [19] (a weaker version is proved in [17]): for any $\epsilon, \epsilon^{\prime} \in(0,1 / 2)$ we have

$$
L_{\epsilon}(p) \asymp L_{\epsilon^{\prime}}(p),
$$

where $f \asymp g$ means that the ratio between the functions $f$ and $g$ is bounded away from 0 and $\infty$ as $p \rightarrow 1 / 2$. In view of this, we fix some $\epsilon \in(0,1 / 2)$ and work with this choice of $\epsilon$ throughout the rest of the paper without loss of generality. We will also need the following five results. The first is a consequence of Theorem 26 of [19] (see also Theorem 1 of [17] for a similar result).

Lemma 2.1 Consider percolation on $\mathcal{T}_{\delta}$ with parameter $p$ and let $C^{w}(r, R)$ (resp., $\left.C^{b}(r, R)\right)$ be the event that the annulus $A(r, R)$ is crossed (from the inner to the outer 
boundary) by a white (resp., black) path. Then,

$$
P_{\delta, p}\left(C^{w}(r, R)\right)=P_{\delta, p}\left(C^{b}(r, R)\right) \asymp P_{\delta, 1 / 2}\left(C^{b}(r, R)\right)=P_{\delta, 1 / 2}\left(C^{w}(r, R)\right)
$$

uniformly in $p$ and $0<r \leq R \leq \delta L_{\epsilon}(p)$.

We interpret this result as follows: on a scale not larger than the correlation length, percolation with parameter $p$ looks roughly like critical percolation.

The second result, stated below, is Remark 38 of [19].

Lemma 2.2 Consider percolation on $\mathcal{T}_{\delta}$ with parameter $p \geq 1 / 2$. Let $C_{H}([0, n] \times[0, k n])$ denote the event that the parallelogram $[0, n] \times[0, k n]$ contains a black horizontal crossing. For any $k \geq 1$ there exist two constants $C_{1}<\infty$ and $C_{2}>0$, both depending on $k$ and $\epsilon$, such that

$$
P_{\delta, p}\left(C_{H}([0, n] \times[0, k n])\right) \leq C_{1} \exp \left(-\frac{C_{2} n}{\delta L_{\epsilon}(p)}\right)
$$

The third result is as follows (see, e.g., $[4,19]$ for more explanation and references).

Lemma 2.3 Consider percolation on $\mathcal{T}_{\delta}$ with parameter $p \geq 1 / 2$, and let

$$
D_{r}=\{\exists \text { black circuit } S \text { surrounding the origin with } \operatorname{diam}(S) \geq r\} .
$$

Then, for each $\epsilon \in(0,1 / 2)$ there exist two constants $C_{3}=C_{3}(\epsilon)<\infty$ and $C_{4}=C_{4}(\epsilon)>0$ such that

$$
P_{\delta, p}\left(D_{r}\right) \leq C_{3} \exp \left(-\frac{C_{4} r}{\delta L_{\epsilon}(p)}\right)
$$

The fourth result is an immediate consequence of Lemma 2.2 (see Fig. 3 for an example of a similar argument).

Lemma 2.4 Consider percolation on $\mathcal{T}_{\delta}$ with parameter $p \geq 1 / 2$, and let

$$
D_{r}^{\prime}=\{\exists \text { black path containing the origin and of diameter at least } r\} .
$$

Fig. 3 The annulus $A\left(x ; r_{1}, r_{2}\right)$ contains four rectangles of side lengths $\left(r_{2}-r_{1}\right) / 2$ and $r_{2}$. If a curve $\gamma$ crosses the annulus $A\left(x ; r_{1}, r_{2}\right)$ then one (indicated with the heavy lines) of the four rectangles contains a crossing in the "easy" direction

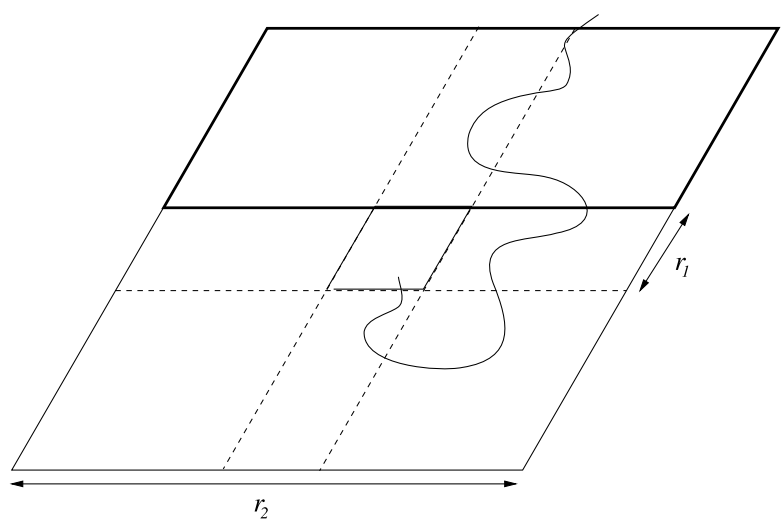


For each $\epsilon \in(0,1 / 2)$ there exist two constants $C_{5}=C_{5}(\epsilon)<\infty$ and $C_{6}=C_{6}(\epsilon)>0$ such that

$$
P_{\delta, p}\left(D_{r}^{\prime}\right) \leq C_{5} \exp \left(-\frac{C_{6} r}{\delta L_{\epsilon}(p)}\right) .
$$

The last result is the celebrated power law for the correlation length [24] (see also [19]): as $p \rightarrow 1 / 2$,

$$
L_{\epsilon}(p)=|p-1 / 2|^{-4 / 3+o(1)} \text {. }
$$

\section{The Scaling Limit}

We turn our attention to the main object of study in this article - the scaling limit of the collection of all boundary loops. We will follow the approach of [10], using the topology introduced in [2].

When taking the scaling limit as the lattice spacing $\delta \rightarrow 0$ one can focus on fixed finite regions, $\Lambda \subset \mathbb{R}^{2}$, or consider the whole $\mathbb{R}^{2}$ at once. The second option avoids dealing with boundary conditions, but requires an appropriate choice of metric. A convenient way of dealing with the whole $\mathbb{R}^{2}$ is to replace the Euclidean metric with a distance function $\Delta(\cdot, \cdot)$ defined on $\mathbb{R}^{2} \times \mathbb{R}^{2}$ by

$$
\Delta(u, v):=\inf _{\varphi} \int\left(1+|\varphi(s)|^{2}\right)^{-1} d s,
$$

where the infimum is over all smooth curves $\varphi(s)$ joining $u$ with $v$, parametrized by arclength $s$, and where $|\cdot|$ denotes the Euclidean norm. This metric is equivalent to the Euclidean metric in bounded regions, but it has the advantage of making $\mathbb{R}^{2}$ precompact. Adding a single point at infinity yields the compact space $\dot{\mathbb{R}}^{2}$ which is isometric, via stereographic projection, to the two-dimensional sphere.

In dealing with the scaling limit we use the approach of Aizenman-Burchard [2]. We regard curves as equivalence classes of continuous functions from the unit interval to $\dot{\mathbb{R}}^{2}$, modulo monotonic reparametrizations. Below, $\gamma$ will represent a particular curve and $\gamma(t)$ a parametrization of $\gamma$. Denote by $\mathcal{S}$ the complete separable metric space of curves in $\dot{\mathbb{R}}^{2}$ with the distance

$$
\mathrm{D}\left(\gamma_{1}, \gamma_{2}\right):=\inf \sup _{t \in[0,1]} \Delta\left(\gamma_{1}(t), \gamma_{2}(t)\right)
$$

where the infimum is over all choices of parametrizations of $\gamma_{1}$ and $\gamma_{2}$ from the interval $[0,1]$. A set of curves (more precisely, a closed subset of $\mathcal{S}$ ) will be denoted by $\mathcal{F}$. The distance between two closed sets of curves is defined by the induced Hausdorff metric as follows:

$$
\operatorname{Dist}\left(\mathcal{F}, \mathcal{F}^{\prime}\right) \leq \varepsilon \quad \Longleftrightarrow \quad\left(\forall \gamma \in \mathcal{F}, \exists \gamma^{\prime} \in \mathcal{F}^{\prime} \text { with } \mathrm{D}\left(\gamma, \gamma^{\prime}\right) \leq \varepsilon \text { and vice versa }\right) .
$$

The space $\Omega$ of closed sets of $\mathcal{S}$ (i.e., collections of curves in $\dot{\mathbb{R}}^{2}$ ) with the metric (4) is also a complete separable metric space. We denote by $\mathcal{B}$ its Borel $\sigma$-algebra.

When we talk about convergence in distribution of random curves, we always mean with respect to the uniform metric (3), while when we deal with closed collections of curves, we always refer to the metric (4). In this paper, the space $\Omega$ of closed sets of $\mathcal{S}$ is used for collections of boundary loops and their scaling limits. 
Aizenman and Burchard [2] formulate a hypothesis that implies, for every sequence $\delta_{j} \downarrow 0$, the existence of a scaling limit along some subsequence $\left\{\delta_{j_{i}}\right\}$. The hypothesis in [2] is formulated in terms of crossings of spherical annuli, but one can work with the annuli defined in Sect. 2 just as well. In order to state it, we need one more piece of notation. For $\delta>0$, we denote by $\mu_{\delta}$ any probability measure supported on collections of curves that are polygonal paths on the edges of the hexagonal lattice $\mathcal{H}_{\delta}$.

In our context, the hypothesis is as follows.

Hypothesis 3.1 For all $k<\infty$ and for all annuli $A(x ; r, R)$ with $\delta \leq r \leq R \leq 1$, the following bound holds uniformly in $\delta$ :

$$
\mu_{\delta}(A(x ; r, R) \text { is crossed by } k \text { disjoint curves }) \leq K_{k}\left(\frac{r}{R}\right)^{\phi(k)}
$$

for some $K_{k}<\infty$ and $\phi(k) \rightarrow \infty$ as $k \rightarrow \infty$.

The next theorem follows from a more general result proved in [2].

Theorem 3.2 ([2]) Hypothesis 3.1 implies that for any sequence $\delta_{j} \downarrow 0$, there exist a subsequence $\left\{\delta_{j_{i}}\right\}_{i \in \mathbb{N}}$ and a probability measure $\mu$ on $\Omega$ such that $\mu_{\delta_{j_{i}}}$ converges weakly to $\mu$ as $i \rightarrow \infty$.

It was already remarked in the appendix of [2] that the above hypothesis can be verified for two-dimensional critical and near-critical percolation. The same conclusion follows from results in [19], and is obtained in Proposition 1 of [20]. We will need a slightly more general result, stated and proved below for completeness.

Lemma 3.3 Let $\left\{\mu_{\delta_{j}, p_{j}}\right\}_{j \in \mathbb{N}}$ be a sequence of measures on boundary paths induced by percolation on $\mathcal{T}_{\delta_{j}}$ with parameters $p_{j}$. For any sequence $\delta_{j} \rightarrow 0$ and any choice of the collection $\left\{p_{j}\right\}_{j \in \mathbb{N}}$, Hypothesis 3.1 holds.

Proof First of all, observe that the number of boundary paths crossing an annulus is necessarily even and that, if there are $k$ disjoint boundary paths crossing the annulus, then the annulus must also be crossed by $k / 2$ disjoint black paths. For any $\delta>0$ and $p \geq 1 / 2$, we have

$$
\begin{aligned}
& P_{\delta, p}(A(x ; r, R) \text { is crossed by } k / 2 \text { disjoint black paths }) \\
& \quad \leq P_{\delta, 1 / 2}(A(x ; r, R) \text { is crossed by } k / 2 \text { disjoint black paths }) \\
& \quad \leq P_{\delta, 1 / 2}(A(x ; r, R) \text { is crossed by a black path })^{k / 2},
\end{aligned}
$$

where we have used monotonicity and the BK inequality [3]. Define $l_{1}, l_{2}$ as the largest, respectively smallest integer such that $1 / 2^{l_{1}} \geq r$, resp. $1 / 2^{l_{2}} \leq R$. Consider the annuli

$$
\begin{aligned}
& A_{1}=A\left(x ;\left(1 / 2^{l_{1}}, 1 / 2^{l_{1}-1}\right), \quad A_{2}=A\left(x ; 1 / 2^{l_{1}-1}, 1 / 2^{l_{1}-2}\right),\right. \\
& A_{N}=A\left(x ; 1 / 2^{l_{2}+1}, 1 / 2^{l_{2}}\right),
\end{aligned}
$$


where $N$ denotes the maximal number of annuli of this type that can be placed in $A(x ; r, R)$. Note that $N$ is of order $\log (R / r)$ and hence there exists a constant $C>0$, independent of $r$ and $R$, such that $\lfloor N / 2\rfloor \geq C \log (R / r)$. Observe furthermore that if $A(x ; r, R)$ is crossed by a black path then none of the annuli $A_{1}, \ldots, A_{N}$ contains a white circuit surrounding $x$. It follows from the RSW theorem (see, e.g., $[15,16,19])$ that the probability of the event that the annulus $A_{i}$ contains a white circuit is uniformly (in $i$ ) bounded from below by some $\gamma>0$, independent of $\delta$. By definition of the annuli, $A_{2 i+1}$ and $A_{2 i^{\prime}+1}$ are disjoint for $i \neq i^{\prime}$. Putting everything together we obtain

$$
\begin{aligned}
& P_{\delta, 1 / 2}(A(x ; r, R) \text { is crossed by a black path })^{k / 2} \\
& \leq P_{\delta, 1 / 2}\left(\bigcap_{i=0}^{\lfloor N / 2\rfloor-1}\left\{A_{2 i+1} \text { does not contain a white circuit surrounding } x\right\}\right)^{k / 2} \\
& =\left[\prod_{i=0}^{\lfloor N / 2\rfloor-1} P_{\delta, 1 / 2}\left(A_{2 i+1} \text { does not contain a white circuit surrounding } x\right)\right]^{k / 2} \\
& \leq\left[(1-\gamma)^{\lfloor N / 2\rfloor}\right]^{k / 2} \\
& \leq\left[(1-\gamma)^{C \log (R / r)}\right]^{k / 2} \\
& =\left[(r / R)^{-C \log (1-\gamma)}\right]^{k / 2} .
\end{aligned}
$$

Therefore, taking $K_{k}=1$ and $\phi(k)=-C \log (1-\gamma) k / 2$, we obtain a bound of the desired form since $-C \log (1-\gamma)>0$.

For any $p \leq 1 / 2$, the same uniform bound follows from swapping white and black in the above argument. Hence, we have the desired bound for any sequence $\delta_{j} \rightarrow 0$ and any $\left\{p_{j}\right\}_{j \in \mathbb{N}}$ and the lemma is proved.

\section{Proof of Theorem 1.1}

We first show how assuming different behaviors for the correlation length leads to the three scenarios described in the theorem. Later we will prove that those three scenarios are nonvoid and are the only three possibilities.

(1) Suppose that for some $\epsilon \in(0,1 / 2), \delta_{j} L_{\epsilon}\left(p_{j}\right) \rightarrow 0$ as $j \rightarrow \infty$. Recall that $\mu$ is the weak limit of a sequence $\left\{\mu_{\delta_{j}, p_{j}}\right\}$ with $\delta_{j} \rightarrow 0$ as $j \rightarrow \infty$ and $p_{j} \geq 1 / 2$. Note that it is actually the case that $p_{j}>1 / 2$ for all but finitely many $j$ since $\delta_{j} L_{\epsilon}\left(p_{j}\right) \rightarrow 0$.

The existence of a boundary loop with positive diameter would imply that there exist $x \in \mathbb{Q}^{2}$ and $0<r_{1}<r_{2} \in \mathbb{Q}$ such that the loop intersects both $B^{o}\left(x, r_{1}\right)$ and $\mathbb{R}^{2} \backslash B\left(x, r_{2}\right)$, so that the annulus $A\left(x ; r_{1}, r_{2}\right)$ is crossed by a boundary path. One of the four (overlapping) parallelograms with side-lengths $\left(r_{2}-r_{1}\right) / 2$ and $r_{2}$ depicted in Fig. 3 is then necessarily crossed at least once in the "easy" direction by a boundary path (see Fig. 3). Let $E$ denote such a crossing event. More precisely, crossings that realize $E$ start and end outside the parallelogram and do not intersect the short sides of the parallelogram. This makes $E$ open in our topology. Note also that the occurrence of $E$ implies, for $\delta_{j}>0$, that the parallelogram contains a black crossing in the easy direction. Thus, the portmanteau theorem and 
Lemma 2.2 yield

$$
\begin{aligned}
\mu\left(A\left(x ; r_{1}, r_{2}\right) \text { is crossed by a boundary path }\right) & \leq 4 \mu(E) \\
& \leq 4 \liminf _{j \rightarrow \infty} \mu_{\delta_{j}, p_{j}}(E) \\
& \leq \liminf _{j \rightarrow \infty} C_{1} \exp \left(-\frac{C_{2}\left(r_{2}-r_{1}\right)}{2 \delta_{j} L_{\epsilon}\left(p_{j}\right)}\right)=0 .
\end{aligned}
$$

We can then conclude that

$$
\begin{aligned}
& \mu(\text { there exists a boundary loop with positive diameter }) \\
& \leq \bigcup_{x \in \mathbb{Q}^{2} ; r_{1}, r_{2} \in \mathbb{Q}^{+}} \mu\left(A\left(x ; r_{1}, r_{2}\right) \text { is crossed by a boundary path }\right)=0 .
\end{aligned}
$$

(2) Suppose that for some $\epsilon \in(0,1 / 2), \delta_{j} L_{\epsilon}\left(p_{j}\right) \rightarrow \infty$ as $j \rightarrow \infty$. Then $1 \leq \delta_{j} L_{\epsilon}\left(p_{j}\right)$ for each $j$ sufficiently large. To show the a.s. existence of an infinite sequence of boundary loops with radii going to zero around the origin (or any other deterministic point), we proceed as follows. Consider the sequence of annuli $A_{1}=A\left(\frac{1}{2}, 1\right), A_{2}=A\left(\frac{1}{4}, \frac{1}{2}\right), \ldots, A_{k}=$ $A\left(\left(\frac{1}{2}\right)^{k},\left(\frac{1}{2}\right)^{k-1}\right), \ldots$, and denote by $F_{k}$ the event that there is (at least) one boundary loop surrounding the origin in the annulus $A_{2 k+1}$ with $k \geq 0$. Since we defined annuli to be closed sets, the event $F_{k}$ is closed in our topology. Note that in order to guarantee the presence of a boundary loop inside the annulus $A_{k}$, it suffices to have, for example, a white circuit in $A\left(\frac{5}{3}\left(\frac{1}{2}\right)^{k},\left(\frac{1}{2}\right)^{k-1}\right)$ and a black circuit in $A\left(\left(\frac{1}{2}\right)^{k}, \frac{1}{3}\left(\frac{1}{2}\right)^{k-2}\right)$ (note that those two annuli are disjoint). Since $\delta_{j} L_{\epsilon}\left(p_{j}\right) \geq 1$ for each large $j$, it follows from Lemma 2.1 and the RSW theorem that the probability to find a white circuit in $A\left(\frac{5}{3}\left(\frac{1}{2}\right)^{k},\left(\frac{1}{2}\right)^{k-1}\right)$ and a black circuit in $A\left(\left(\frac{1}{2}\right)^{k}, \frac{1}{3}\left(\frac{1}{2}\right)^{k-2}\right)$ is bounded away from 0 as $j \rightarrow \infty$, uniformly in $k$. Therefore there exists $\varepsilon_{0}>0$ such that

$$
\mu\left(F_{k}\right) \geq \limsup _{j \rightarrow \infty} \mu_{\delta_{j}, p_{j}}\left(F_{k}\right) \geq \varepsilon_{0}, \quad \text { for every } k,
$$

where the first inequality follows from the portmanteau theorem. Note also that the events $F_{k}$ and $F_{k^{\prime}}$ are independent for $k^{\prime} \neq k$. Moreover, $\sum_{k=0}^{\infty} \mu\left(F_{k}\right)=\infty$ and thus by the BorelCantelli lemma there are infinitely many boundary circuits surrounding the origin with diameter going to zero, $\mu$-a.s.

We argue in a similar way as above to show that every point is surrounded by a countably infinite family of nested loops with radii going to infinity. Let $B_{k}$ denote the annulus $A\left(2^{k}, 2^{k+1}\right)$ and write $F_{k}^{\prime}$, with $k \geq 0$, for the event that the annulus $B_{2 k+1}$ contains at least one boundary loop surrounding $B(1)$. For each $k$ it holds that $\delta_{j} L_{\epsilon}\left(p_{j}\right) \geq 2^{k+1}$ for $j$ sufficiently large. Hence, it follows from Lemma 2.1 and the RSW theorem that the probability to find a boundary loop in $B_{2 k+1}$ is uniformly (in $k$ ) bounded away from 0 as $j \rightarrow \infty$. Again, the event $F_{k}^{\prime}$ is closed in our topology, thus $\mu\left(F_{k}^{\prime}\right) \geq \limsup _{j \rightarrow \infty} \mu_{\delta_{j}, p_{j}}\left(F_{k}^{\prime}\right) \geq \varepsilon_{1}$, for some $\varepsilon_{1}>0$ independent of $k$. Hence, the Borel-Cantelli lemma implies that there are infinitely many boundary loops surrounding $B(1)$ with diameter going to infinity $\mu$-a.s. By translation invariance, the same is true for every $B(x ; 1)$ with $x \in \mathbb{Q}$ and therefore for every point of the plane.

(3) Suppose that for some $\epsilon \in(0,1 / 2), \delta_{j} L_{\epsilon}\left(p_{j}\right)$ stays bounded away from both 0 and $\infty$ as $j \rightarrow \infty$. That is, there exist $\beta>0$ and $K<\infty$ such that $\beta \leq \delta_{j} L_{\epsilon}\left(p_{j}\right) \leq K$ for each $j$ sufficiently large. The first part of the proof in case (2) carries over directly to the present case, with 1 replaced by $\beta$ in the lower bound for the macroscopic correlation length and 
the annuli $A\left(1 / 2^{k}, 1 / 2^{k-1}\right)$ replaced by $A\left(\beta / 2^{k}, \beta / 2^{k-1}\right)$. Thus, $\mu$-a.s. there exist infinitely many boundary loops surrounding the origin, with diameter going to zero.

Our next goal is to prove the a.s. existence of a largest boundary loop surrounding the origin. Let $G_{L}$ denote the event that there exists a largest boundary loop $\gamma$ surrounding or containing the origin and that this loop has $\operatorname{diam}(\gamma) \leq L$. Then $G:=\bigcup_{L=1}^{\infty} G_{L}$ is the event that there exists a largest loop surrounding or containing the origin. Note that if all black circuits around the origin have diameter smaller than $L$ and there is no black path containing the origin of diameter larger than $L-2 \delta_{j}$, then $G_{L}$ occurs. Therefore $\mu_{\delta_{j}, p_{j}}\left(G_{L}\right) \geq 1-$ $\left[P_{\delta_{j}, p_{j}}\left(D_{L}\right)+P_{\delta_{j}, p_{j}}\left(D_{L-2 \delta_{j}}^{\prime}\right)\right]$, where $D_{L}$ is the event that the origin is surrounded by a black circuit of diameter at least $L$ and $D_{L-2 \delta_{j}}^{\prime}$ is the event that there is a black path containing the origin of diameter at least $L-2 \delta_{j}$. Using Lemmas 2.3 and 2.4 and the fact that the event $G_{L}$ is closed in our topology, we can write

$$
\begin{aligned}
\mu\left(G_{L}\right) & \geq \limsup _{j \rightarrow \infty} \mu_{\delta_{j}, p_{j}}\left(G_{L}\right) \\
& \geq 1-\liminf _{j \rightarrow \infty}\left[P_{\delta_{j}, p_{j}}\left(D_{L}\right)+P_{\delta_{j}, p_{j}}\left(D_{L-2 \delta_{j}}^{\prime}\right)\right] \\
& \geq 1-\liminf _{j \rightarrow \infty} C^{\prime} \exp \left(-\frac{C^{\prime \prime} L}{\delta_{j} L_{\epsilon}\left(p_{j}\right)}\right) \\
& \geq 1-C^{\prime} \exp \left(-\frac{C^{\prime \prime} L}{K}\right) .
\end{aligned}
$$

Since the events are nested (i.e., $G_{L_{1}} \subset G_{L_{2}}$ for $L_{1}<L_{2}$ ),

$$
\mu(G)=\lim _{L \rightarrow \infty} \mu\left(G_{L}\right) \geq \lim _{L \rightarrow \infty}\left[1-C^{\prime} \exp \left(-\frac{C^{\prime \prime} L}{K}\right)\right]=1 .
$$

Since boundary loops cannot cross each other and, by the previous part of the proof, the origin is surrounded with probability one by a sequence of infinitely many boundary loops with diameter going to zero, the largest boundary loop does not touch the origin. Hence, the event $G$ coincides with the existence of a largest boundary circuit surrounding the origin and we are done.

To continue the proof, note that for each $\epsilon \in(0,1 / 2)$, as $j \rightarrow \infty$,

- either $\delta_{j} L_{\epsilon}\left(p_{j}\right) \rightarrow 0$,

- or $\delta_{j} L_{\epsilon}\left(p_{j}\right) \rightarrow \infty$,

- or $\delta_{j} L_{\epsilon}\left(p_{j}\right)$ is bounded away from both 0 and $\infty$.

This is clearly so because we are assuming that $\left\{\mu_{\delta_{j}, p_{j}}\right\}_{j \in \mathbb{N}}$ has a limit $\mu$, and we have proved that the three cases above give rise to three incompatible scenarios for $\mu$. Indeed, if we are not in one of the three cases above, then there must be two different subsequences of $\left\{\left(\delta_{j}, p_{j}\right)\right\}_{j \in \mathbb{N}}$ falling in two different cases, which contradicts the existence of a limit $\mu$. We can then conclude that there are no other possible scenarios for $\mu$ besides the three described in the theorem.

To conclude the proof, we need to show that all three scenarios are non-void. For the first two, this is obvious. To prove that the third scenario is also non-void, take $0<\epsilon_{1}<\epsilon_{2}<1 / 2$ and consider any sequence $\left\{\left(\delta_{j}, p_{j}\right)\right\}_{j \in \mathbb{N}}$ such that $\delta_{j} \rightarrow 0$ and $p_{\epsilon_{1}}^{+}\left(1 / \delta_{j}\right) \leq p_{j} \leq p_{\epsilon_{2}}^{+}\left(1 / \delta_{j}\right)$. This implies that $L_{\epsilon_{1}}\left(p_{j}\right) \leq 1 / \delta_{j} \leq L_{\epsilon_{2}}\left(p_{j}\right)$ for each $j$. We can assume without loss of generality that the sequence $\left\{\mu_{\delta_{j}, p_{j}}\right\}_{j \in \mathbb{N}}$ has a limit $\mu$. (If that is not the case, by Theorem 3.2 
and Lemma 3.3 we can extract a subsequence $\left\{\mu_{\delta_{j_{k}}, p_{j_{k}}}\right\}_{k \in \mathbb{N}}$ that does have a limit, and rename it $\left\{\mu_{\delta_{j}, p_{j}}\right\}_{j \in \mathbb{N}}$.) Since $\delta_{j} L_{\epsilon_{1}}\left(p_{j}\right)$ remains bounded as $j \rightarrow \infty, \delta_{j} L_{\epsilon}\left(p_{j}\right)$ must remain bounded as $j \rightarrow \infty$ for every other $\epsilon \in(0,1 / 2)$ because $L_{\epsilon}(p) \asymp L_{\epsilon_{1}}(p)$. Analogously, since $\delta_{j} L_{\epsilon_{2}}\left(p_{j}\right)$ is bounded away from 0 as $j \rightarrow \infty, \delta_{j} L_{\epsilon}\left(p_{j}\right)$ must be bounded away from 0 as $j \rightarrow \infty$ for every $\epsilon \in(0,1 / 2)$. Therefore, for each $\epsilon \in(0,1 / 2), \delta_{j} L_{\epsilon}\left(p_{j}\right)$ remains bounded away from both 0 and $\infty$ as $j \rightarrow \infty$, showing that $\mu$ falls in the third scenario.

Remark 4.1 A significantly stronger version of case (2) can be proven, namely that $\mu$ in this case coincides with the full scaling limit of critical percolation. In order to prove this one can use the same strategy as in [10], combined with the proof of Proposition 4 of [20]. Below we briefly sketch how one can obtain the result by modifying the arguments of [10]. We stress that this is not meant to be a self-contained proof, and some familiarity with [10] is needed in order to follow the arguments outlined below.

First of all, wherever statement (S) (concerning the convergence of the critical exploration path to $\mathrm{SLE}_{6}$ - see p. 18 of [10]) is invoked in [10], one needs to use the proof of Proposition 4 of [20]. We remark that the statement of Proposition 4 of [20] concerns triangular domains and is therefore not sufficient for our purposes, but the proof applies in much greater generality. In particular, the reader can check that it applies to the situations that arise in [10].

Uniform bounds on "six-arm" events in the plane and "three-arm" events near a boundary (see Lemma 6.1 of [10] and its proof) are used repeatedly in [10]. Thanks to Theorem 1 of [17] and similar results described in [19] (see, e.g., Sect. 3.2, and Theorem 27 and the discussion following it), such uniform bounds are also available for percolation with parameter $p_{j}$ on $\mathcal{T}_{\delta_{j}}$ inside a disc of diameter $L$, provided that $\delta_{j} L_{\epsilon}\left(p_{j}\right) \geq L$. Due to the assumption that $\delta_{j} L_{\epsilon}\left(p_{j}\right) \rightarrow \infty$ as $j \rightarrow \infty$, such a condition is satisfied for any $L<\infty$, for $j$ sufficiently large.

Some care is also needed in the proof of the second part of Theorem 5 of [10] (see p. 19 for the statement; the proof begins on p. 27), and in particular of Lemma 6.4 (see p. 27) and Lemma 6.6 (see p. 29) used in that proof. The proof of the second part of Theorem 5 of [10] is given for critical percolation, but the only features of critical percolation that are really used are uniform bounds on certain crossing probabilities (involving crossings of a rectangle or an annulus). Due to the above considerations, similar bounds can be used in the present context (see again Sect. 3.2 and Theorem 27 of [19]).

Acknowledgement The first author thanks C.M. Newman for a useful discussion.

Open Access This article is distributed under the terms of the Creative Commons Attribution Noncommercial License which permits any noncommercial use, distribution, and reproduction in any medium, provided the original author(s) and source are credited.

\section{References}

1. Aizenman, M.: Scaling limit for the incipient spanning clusters. In: Mathematics of Multiscale Materials. IMA Vol. Math. Appl., vol. 99, pp. 1-24. Springer, New York (1998)

2. Aizenman, M., Burchard, A.: Hölder regularity and dimension bounds for random curves. Duke Math. J. 99, 419-453 (1999)

3. van den Berg, J., Kesten, H.: Inequalities with applications to percolation and reliability. J. Appl. Probab. 22, 556-569 (1985)

4. van den Berg, J., Peres, Y., Sidoravicius, V., Vares, M.E.: Random spatial growth with paralyzing obstacles. Ann. Inst. Henri Poincaré Probab. Stat. 44, 1173-1187 (2008) 
5. Bollobas, B., Riordan, O.: Percolation. Cambridge University Press, Cambridge (2006)

6. Borgs, C., Chayes, J., Kesten, H., Spencer, J.: The birth of the infinite cluster: finite-size scaling in percolation. Commun. Math. Phys. 224, 153-204 (2001)

7. Camia, F., Fontes, L.R.G., Newman, C.M.: The scaling limit geometry of near-critical 2D percolation. J. Stat. Phys. 125, 1155-1171 (2006)

8. Camia, F., Fontes, L.R.G., Newman, C.M.: Two-dimensional scaling limits via marked nonsimple loops. Bull. Braz. Math. Soc. 37, 537-559 (2006)

9. Camia, F., Newman, C.M.: Continuum nonsimple loops and 2D critical percolation. J. Stat. Phys. 116, 157-173 (2004)

10. Camia, F., Newman, C.M.: Two-dimensional critical percolation: the full scaling limit. Commun. Math. Phys. 268, 1-38 (2006)

11. Camia, F., Newman, C.M.: Critical percolation exploration path and $\mathrm{SLE}_{6}$ : a proof of convergence. Probab. Theory Relat. Fields 139, 473-519 (2007)

12. Chayes, J.T., Chayes, L., Fisher, D., Spencer, T.: Finite-size scaling and correlation lengths for disordered systems. Phys. Rev. Lett. 57, 2999-3002 (1986)

13. Garban, C.: Processus SLE et Sensibilité aux Perturbations de la Percolation Critique Plane. Doctoral Thesis, Université Paris Sud (2008)

14. Garban, C., Pete, G., Schramm, O. (in preparation)

15. Grimmett, G.R.: Percolation, 2nd edn. Springer, Berlin (1999)

16. Kesten, H.: Percolation Theory for Mathematicians. Birkhäuser, Boston (1982)

17. Kesten, H.: Scaling relations for 2D-percolation. Commun. Math. Phys. 105, 109-156 (1987)

18. Lawler, G., Schramm, O., Werner, W.: One arm exponent for critical 2D percolation. Electron. J. Probab. 7(2), 1-13 (2002)

19. Nolin, P.: Near-critical percolation in two dimensions. Electron. J. Probab. 13, 1562-6123 (2008)

20. Nolin, P., Werner, W.: Asymmetry of near-critical percolation interfaces. J. Am. Math. Soc. 22, 797-819 (2008)

21. Schramm, O.: Scaling limits of loop-erased random walks and uniform spanning trees. Isr. J. Math. 118, 221-288 (2000)

22. Smirnov, S.: Critical percolation in the plane: Conformal invariance, Cardy's formula, scaling limits. C. R. Acad. Sci. Paris 333, 239-244 (2001)

23. Smirnov, S.: Towards conformal invariance of 2D lattice models. In: International Congress of Mathematicians, vol. II, pp. 1421-1451. Eur. Math. Soc., Zürich (2006)

24. Smirnov, S., Werner, W.: Critical exponents for two-dimensional percolation. Math. Rev. Lett. 8, 729744 (2001) 